\title{
STRATÉGIES ESTHÉTIQUES DE L'ORGANISATION ET PRAGMATIQUE DU SENSIBLE
}

\section{Philippe Quinton'}

La réflexion proposée ici s'appuie sur des recherches de terrain et des pratiques professionnelles. Il s'agit de cerner quelques objets et enjeux communicationnels spécifiques à la dimension sensible dans et des organisations, mais aussi préciser ce qui peut en être saisi par les sciences de l'information et de la communication. Il semble en effet utile de restituer à l'esthétique -comme objet de recherche interdisciplinaire - la place qui lui revient au sein des Sic, alors qu'elles semblent la tenir à distance ${ }^{2}$. L'esthétique n'étant pas qu'un adjuvant artistique ou cosmétique à la communication, on postulera que c'est l'une de ses dimensions relationnelles nodales, elle fait corps avec le

1 Université Pierre Mendès-France - Grenoble II et Centre d'étude de l'écriture (Paris VII-CNRS).

2 Il est vrai que, comme pour la création, une bonne partie de la dimension esthétique semble insaisissable par la science. Mais les Sic gagneraient à s'approprier davantage les aspects de ce domaine qui les concernent. Le sensible, comme le sensoriel, est omniprésent dans les processus d'information et de communication, il mérite des protocoles d'analyse spécifiques, à condition d'oublier les réticences dues à ses affinités artistiques qui engagent méfiance et doute épistémologique à son égard.

Recherches en communication, $\mathrm{n}^{\circ} 17$ (2002). 
sémiotique, le technique et le social, même banalisée par l'économique.

À l'évidence le beau n'est pas une problématique fondamentale des organisations ${ }^{1}$, sauf pour celles dont c'est l'objet social ou l'outil ${ }^{2}$; elles ne recherchent pas le sublime, la grâce ou un absolu désintéressé mais la réalisation efficace de leurs objectifs (service, mission, profit...), ce qui nécessite quand même une attention à la forme, aux présentations et représentations des choses. Il est dit qu'elles veulent séduire, convaincre, plaire, émouvoir, créer du désir et de l'intérêt autour d'un produit ou d'une marque pour exister, impliquer leurs clients et vendre. L'approche sensible serait alors une stratégie d'image comme une autre, une des modalités d'argumentation et d'existence de la marque. Le principe de réalité domine largement le principe de plaisir et la jouissance esthétique n'a apparemment pas sa place dans ces univers rationnels. Mais depuis bien longtemps, les rapports sensibles entre les êtres et les choses sont constitutifs des interactions et des représentations humaines. Situés au cœur des processus d'interprétation, ils sont assujettis à autant de normes, voire de dogmes, que l'organisation. Ainsi, la dimension esthétique est présente de facto dans toute manifestation sensible, et comme pour l'art contemporain, son refus en est une...

L'expérience esthétique s'entend dès lors comme construit social et phénomène relationnel ; ce n'est pas qu'une affaire de subjectivité artistique. Cela engage deux hypothèses dont le traitement ne sera qu'ébauché ici, faute de place :

- elle est fondamentalement systémique, car le sensible est un système complexe articulé avec d'autres ;

- en tant que mise en acte sensible elle ouvre une dimension pragmatique essentielle à la communication, ce qui invite à y observer les différents rapports entre sensible et intentionnalité, tels qu'ils se construisent en production (avec les designs) et en réception.

1 Ce terme générique désigne des entreprises, institutions, associations, collectivités locales, administrations, etc.

2 Comme les agences de design et de publicité, les entreprises culturelles ou les associations artistiques... 


\section{Les objets de l'esthétique}

La dimension esthétique est apportée par l'humain dans un environnement donné, pas par l'objet qui lui sert de support imaginaire ("cet objet est esthétique"), ni par l'activité qui le forme ("c'est design..."). Elle dépend donc fortement des conditions de perception et des systèmes de valeur en présence, et bien que l'acception classique veuille qu'elle puisse universellement se partager, ce n'est pas forcément le cas pour les organisations.

La production (créative ou pas) mobilise des ressources et génère des expressions sensibles très différentes des impressions sensibles et des interprétations propres à la réception. Dans les deux cas l'esthétique relève de modélisations perceptives et cognitives complexes qui peuvent être identifiées. Jauss ${ }^{1}$ montre que les pratiques esthétiques vont du banal au sublime, et que chacun recherche une participation et une identification particulière dans les objets livrés à son expérience subjective. Avec cette forte subjectivité et des jugements de valeurs très relatifs, les choses ne sont alors pas si simples pour qualifier et évaluer une esthétique de l'entreprise et ses objets. La qualification, l'analyse ou l'évaluation esthétiques ont du mal à échapper au jugement, en l'absence de procédures adaptées et de lexiques adéquats, aussi bien pour les organisations que pour les chercheurs.

On pourrait penser que le plaisir des acteurs de l'organisation n'est acceptable que s'il génère une adhésion, une identification favorable à un profit, ce qui assure à court terme la satisfaction primordiale du client et de l'actionnaire. Tout aménagement ou décision à dominante esthétique serait donc effectué en interne sous la contrainte du management, en externe sous la tutelle du marketing, et de toute manière soumis au contrôle du gestionnaire. L'objet de l'esthétique n'est alors que relatif, réduit à un ensemble de moyens pour une recherche d'effets. Pourtant, les conceptions usuelles et persistantes (héritées de Kant) placent l'esthétique dans un absolu en rapport avec le beau et l'universalité du jugement de goût, ou encore l'harmonie. Les objets esthétiques de l'organisation doivent donc se situer dans ce grand écart épistémologique. Mais s'ils opèrent à l'évidence dans des territoires, des moyens d'identification, des composantes d'une

1 H. R. Jauss, Pour une esthétique de la réception, Paris, Gallimard, 1978. 
identité et plus localement des systèmes de communication situés, ils n'y sont pas enfermés.

Cette question de l'esthétique des organisations concernant des acteurs, des faits et des objets matériels très divers, on se limitera aux designs -en tant que processus et résultats pris comme objets de recherche- et en particulier ceux qui se rapportent aux communications graphiques (identification visuelle, signalétique, éditions papier et web, vidéos, expositions, etc.). Il convient d'abord de s'extraire des débats obsolètes et réducteurs sur art et esthétique ou art et communication, pour observer comment l'esthétique s'accommode et se nourrit de la stratégie, de quelle manière elle se met au service de l'argumentation et de l'influence.

Herman Parret ${ }^{1}$ parle d'une "esthétisation de la pragmatique" en s'intéressant aux "franges esthétiques des discours", ce qui peut nous inviter au passage à considérer le sensible comme une forme de discours, et prêter attention à ses manifestations non-verbales les plus discrètes dans la communication, dans la mesure où elles fournissent des supports et des marges de manœuvres essentielles aux médiations.

Enfin, Jean Caune ${ }^{2}$ précise que l'esthétique ne se confine pas à l'artistique, d'autres objets ou mises en actes peuvent tout aussi bien supporter une relation esthétique et porter des émotions ou des savoirs. Avec lui, on peut déplorer qu'après Kant la limitation "du sens commun au goût et du goût au jugement esthétique" ait été favorisée, ce qui ne peut que réduire nos approches de ces notions. En communication, la dimension esthétique se dilue dans les principes de cohérence, d'identification, d'efficacité, de différence, de pertinence ou d'originalité, etc., plus facilement applicables aux messages de l'organisation. Cette orientation esthétique est partie prenante d'une nouvelle forme d'universalité.

\section{L'universalité esthétique}

L'environnement économique et social est de plus en plus favorable aux "belles choses", de qualité et fonctionnelles (cela sert à quoi ?), mais qui doivent aussi avoir du sens (qu'est-ce que cela veut

1 H. PARret, L'esthétique de la communication. L'au-delà de la pragmatique, Bruxelles, Ousia, 1999.

2 J. CAUnE, Esthétique de la communication, Paris, PUF, coll. "Que sais-je ?", $\mathrm{n}^{\circ} 3259,1997$. 
dire ?) ; car les formes matérielles ne sont plus là pour elles-mêmes. Beaux bureaux, belles voitures, beaux vêtements, beaux magasins, etc., la préoccupation esthétique est commune, c'est un besoin comme un autre qui procède d'un univers d'attentes et fait partie de la vie sociale. Reste à savoir comment cela peut s'évaluer, selon quelles références et quels modèles dans une communauté donnée.

Chacun veut plus de sensible, plus d'émotions, même basiques ou programmées (la Coupe du Monde 98); il s'agit de construire de meilleures relations avec les êtres ou les choses et que ces productions soient lisibles et accessibles. La vie sociale et l'entreprise prennent de la distance par rapport à elles-mêmes en s'esthétisant dans les événements et la plupart de leurs formes discursives. Les organisations soignent les designs de leurs communications ; de leurs environnements matériels, sensoriels et symboliques; de leurs produits et surtout de leurs marques, situées en première ligne du commerce esthétisé.

Pourtant, l'exigence de signification et de plaisir n'empêche pas une certaine accoutumance aux incantations stéréotypées de l'innovation, dans la course effrénée vers le nouveau qui voit beaucoup de signes, d'objets ou d'idées se ressembler (les noms, les marques, les formes, les sites...) dans des approches visuelles semblables, car la culture commerciale tend à homogénéiser un peu plus les segmentations des goûts et des couleurs, comme si la raison marchande finissait toujours par contrôler le sensible, préconstruire l'idée du beau et renforcer les usages établis.

Toutefois, la pression esthétique stimule les productions et les médiations organisationnelles. Le beau est devenu stratégique, instrument de conquête de marchés -et marché lui-même-, gage de notoriété. Les grands registres de communication : entreprise, institution, produit et marque ${ }^{1}$ sont donc tenus de prendre en compte cette injonction esthétique moderne.

Selon Jauss, l'expérience esthétique est une "fonction de l'activité humaine"2. Les concepts classiques de la tradition esthétique (poiesis, aisthésis et catharsis), qu'il mentionne à propos de l'art, peuvent s'appliquer aux organisations, qui en tant que sociétés humaines, en ont aussi besoin. Ainsi, la poiesis relève d'une pratique qui permet de participer au monde, de manifester une appartenance et

$1 \mathrm{Ph}$. Schwebig, Les communications de l'entreprise, Paris, Mac Graw-Hill, 1988.

2 H. R. JAuss, op. cit. 
une appropriation sensible; l'aisthésis régénère la perception esthétique et intuitive des choses ; la catharsis comme processus d'identification libère des contraintes et facilite l'intégration sociale. À tous les niveaux, la relation est donc au centre de cette question esthétique.

\section{L'esthétique comme relation}

À travers son esthétique de la communication Fred Forest ${ }^{1}$ a montré que la communication pouvait être l'objet de la pratique artistique, l'artiste faisant à l'occasion sa propre publicité. Mais cette jonction art-communication est maintenue dans le champ de l'art. Selon une démarche opposée, se situerait une utilisation de l'art comme support de la communication, ce que font le mécénat et surtout la publicité avec son potentiel connu de recyclage esthétique.

Au-delà de cette dimension communicationnelle de l'expérience esthétique, Jean Caune, situe l'esthétique de la communication là où il y a "une expressivité dans la communication", et quand cette expression est une fonction de communication. Selon lui, cette esthétique "concerne une approche des phénomènes de relation sociale du point de vue du contact et du lien sensible"2.

Pour le chercheur qui s'intéresse à cette relation et à ses contenus spécifiques, il s'agit alors de conduire une analyse sémiopragmatique et ethnosémiotique exigeant une construction d'objets et des formulations spécifiques, afin de comprendre comment et à partir de quoi le destinataire élabore son expérience sensible, ce qu'elle contient (et d'où cela vient) et ce qui en résulte.

Certaines multinationales quittent la logique produit pour créer uniquement une relation dont le seul contenu est constitué des valeurs de la marque. Cette prééminence de la marque sur le produit, signalée autant par ses théoriciens ${ }^{3}$ que par ses critiques ${ }^{4}$, atteste de l'amplitude du marketing symbolique des objets-signes et de l'importance économique des valeurs immatérielles.

1 Fr. Forest, "Manifeste pour une esthétique de la communication", Plus moins zéro, n 43, Bruxelles, 1984.

2 J. CAUNE, op. cit.

3 J.-N. KAPFERER, La marque, Paris, Éd. d'Organisation, 1996 ; A. SEMPRINI, Le marketing de la marque, Paris, Éd. Liaisons, 1992.

4 N. KLEIN, No logo, Leméac/Actes Sud, 2001. 
L'esthétique n'est donc pas une finalité sans fin dans l'organisation puisque ce n'est qu'un moyen de créer des relations, des tensions, des désirs, entre objets et sujets, par les voies sensibles. Cette esthétique-là est construite, raisonnée, avec une dynamique toujours intéressée.

Jean Baudrillard ${ }^{1}$ et Jean-Marie Schaeffer ${ }^{2}$ ont montré chacun à leur manière que l'essentiel est dans la relation qui s'établit avec l'objet, usuel ou artistique, et pas dans l'objet lui même. C'est en effet l'usage esthétique des choses qui mérite intérêt. Ainsi, l'esthétique ne serait pas définissable par des caractéristiques fonctionnelles, structurelles, formelles ou symboliques propres à des objets matériels dans des environnements particuliers ${ }^{3}$, mais à travers leurs mises en relations avec un ou des sujets. L'esthétique serait alors une pragmatique du sensible inscrite au cœur des processus de communication. Dans la même voie, Jean Caune propose de concevoir l'expérience esthétique comme une "activité sensible et intelligible" qui "établit une relation entre un sujet et un objet ou des sujets entre eux"4. Ainsi, cette expérience "inscrit la subjectivité dans la communauté culturelle". L'organisation pense tirer parti de cette possibilité et considère l'esthétique comme un ingrédient ou un adjuvant de l'image, de l'identité ou de la culture, alors que la dimension sensible en est l'expression même. En termes logiques, l'esthétique n'est pas le membre d'une classe (les composantes de la relation) mais la classe elle-même (la relation), et c'est dans ses mises en formes relationnelles (support, message, fonction, discours...) qu'elle offre une prise à une approche systémique permettant d'échapper au jugement de valeur, à la métaphore ou au commentaire, ses principaux errements scientifiques.

\section{L'esthétisation organisationnelle}

Un créateur d'entreprise peut esthétiser sa création et en parler comme de son æuvre; mais cette relation avec sa société n'est pas celle de celui la perçoit de l'extérieur. Il peut manifester très tôt le

1 J. BAUDRILlARD, Le système des objets, Paris, Gallimard, 1968.

2 J.-M. SCHAEFFER, Les célibataires de l'art, Paris, Gallimard, 1996.

$3 \mathrm{Br}$. Borja de Mozota, Design et management, Paris, Éd. d'Organisation, 1990.

4 J. CAUne, Pour une éthique de la médiation. Le sens des pratiques culturelles, Grenoble, Presses universitaires de Grenoble, 1999. 
besoin d'avoir un logotype qui matérialise visuellement l'entreprise en voie d'établissement. Sans forcément procéder d'une intention esthétique, car ce qui compte c'est d'attester une existence, le logo est doté ici de vertus légitimantes. Mais le sentiment d'existence n'est que celui du créateur aux valeurs duquel un groupe va être fermement invité à souscrire. Dans les phases de production, les discours managériaux qualifient les produits d'ceuvre collective, résultant d'un travail ou d'un esprit d'équipe, en vue d'impliquer davantage les individus, d'accentuer leur identification aux objectifs de l'organisation. Ce processus d'esthétisation du collectif qui articule des projets trouve des appuis dans toutes les dimensions visuelles, sonores et tactiles de l'organisation : éditions, vêtements, locaux, outils, formation, procédures, événements, célébrations, discours, etc. Mais les organisations s'achètent, fusionnent ; les acteurs et les cultures sont brassés dans les changements de structures et les montages financiers qui entraînent de facto des affrontements esthétiques dans lesquels les logos accompagnent la vie ou la mort des identités. L'arrivée d'Orange tue Itinéris et Ola. Les esthétiques triomphantes affichent des ambitions universelles, et génèrent des formules graphiques transnationales et aseptisées, à l'opposé des discours de différenciation.

\section{L'esthétique entre jugement et décision}

La commande a son esthétique, une manière d'exprimer des désirs, des stratégies et des projets qui doivent prendre une expression sensible dans une forme graphique. Ses formulations témoignent (à travers un cahier des charges parfois esthétiquement prescriptif) des ambitions d'un programme et des motivations d'un commanditaire. Il est donc utile à ce niveau de s'intéresser de près aux stratégies des acteurs en présence, vu que les organisations sont des communautés d'intérêts contradictoires, avec des jeux de pouvoir subtils entre les humains et leurs signes. J'ai eu l'occasion de montrer comment les logotypes et les dispositifs graphiques servent les stratégies de pouvoir ou les plans de carrière; de quelle manière le design graphique balise la négociation et la conduite des changements ${ }^{1}$.

$1 \mathrm{Ph}$. Quinton, Design graphique et changement, Paris, L'Harmattan, 1997. 
Contrairement au sensus communis de Kant, le jugement esthétique n'est pas la chose la mieux partagée dans l'organisation. Le beau est ce qui plaît au patron ou au groupe dominant, lequel légitime ainsi les valeurs qu'il entend donner à l'organisation et auxquelles les subordonnés sont priés d'adhérer. La décision s'exerce dans un cadre de tensions nécessaires entre management et création, contraintes et idéal, qui plus est lorsqu'il est question de propositions visuelles faites par des créatifs dont le côté un peu "artiste" effraie, dans un cadre de pensée qui confond aisément création et esthétique. On prête à leur fonctionnement intuitif un risque esthétique fictif alors qu'il s'agit d'une démarche spécifique -avec ses méthodologies de recherche et ses concepts systémiques- dont les racines artistiques les portent vers les transformations, les décalages, les transgressions socialement utiles.

Lorsque l'acteur dominant de la commande est confronté aux propositions visuelles des designers, il mobilise ses propres critères de jugement et exprime souvent ses goûts personnels, mâtinés d'une doxa plastique rudimentaire, plus que sa position de manager et ce qu'elle suppose. On ne sait pas évaluer la dimension esthétique au même titre qu'une dimension technique dans les organisations, elle semble d'ailleurs étrangère aux études d'ingénieur ou de management. Ainsi, s'accommode-t-on d'arbitraire et de référents sommaires en matière visuelle alors que les décisions sur les process techniques ou les chiffres obéissent à des évaluations très rationnelles. En quelque sorte, on voudrait que l'esthétique (à travers ses dispositifs sensibles) soit opérationnelle, qu'elle fonctionne, mais on ne lui reconnaît pas cette possibilité rationnelle (son ingénierie propre) alors qu'elle l'a effectivement (ce pourquoi justement on peut la qualifier de systémique).

Décider c'est choisir et s'engager, tous les enjeux organisationnels se catalysent dans cette phase. Mais la confusion entre décision et jugement de valeur, arbitraire et subjectif, convention et certitude, valeur et vérité, brouille les appuis de la décision. Le designer doit faire face à des appréciations banales comme "c'est joli", "c'est beau" ou "c'est moche", "cela ne me plaît pas", qui amènent les tenants du pouvoir effectif de décision ${ }^{1}$ à décider du sort d'une production en

1 Les communicants (mandatés) qui pilotent le processus sont rarement les vrais décisionnaires sur les gros projets. Ils en rendent toujours compte au pdg ou au comité de pilotage seuls habilités à valider une proposition. 
fonction de leurs impressions, de codes sommaires (ex. : les couleurs), ou en se référant à des principes et des répertoires personnels inadaptés au cadre professionnel. Sans parler des nombreux cas où le projet fait les frais des luttes internes dont les objets n'ont rien à voir avec l'esthétique... De la sorte, le goût ou les raisons du commanditaire (individu ou collectif) prévalent souvent sur ceux des destinataires réels d'une production.

Cependant, les concepteurs tendent eux aussi à formater leurs propositions en fonction des critères de jugement ou des goûts de leurs clients. L'important pour eux est que leur projet "passe", ce qui peut les amener à préférer les combinatoires et les effets à la mode aux impertinences créatives des ruptures. Par exemple, les discours d'accompagnement qu'ils produisent sur les logotypes expliquent ce qu'il faut voir ; ils orientent les perceptions de décideurs peu experts en graphisme, mais aussi des utilisateurs et médiateurs des systèmes graphiques proposés. Ainsi, l'argumentation a posteriori sur les signes créés les prémunit contre les futures interprétations inopportunes, l'esthétique est contrôlée par le verbe. Les constats de terrain montrent que les commanditaires, comme les journalistes, reprennent aisément et mot pour mot ces discours construits moteurs des dossiers de presse ${ }^{1}$.

\section{Systèmes esthétiques et stratégies sensibles des designs}

Loin de la quête d'un beau idéal, les designs ${ }^{2}$ sont historiquement issus de l'esthétique industrielle, dont le projet était de rechercher le beau utile dans une production massive d'objets (la "forme utile"), et donc de se débarrasser de l'ornement en renonçant aux Arts décoratifs. Avec le Bauhaus, ce fonctionnalisme a été systématisé et universalisé, la majorité des productions du siècle en ont hérité, mais au prix d'une réduction du design au produit (mobilier, ustensiles...)

1 Ph. QuinTon, "Logo ergo sum", Étapes graphiques, n 65, sept. 2000.

2 Le terme design prend ses racines dans le vieux français : desseigner (intention, projet, volonté) et dessigner (dessiner), cousins du latin designare. L'anglicisme vient de chez nous... Le terme, jamais utilisé seul, qualifie des activités très différentes : design graphique (et ses dérivés: d'interface; interactif; multimédia...) ; design industriel ; design d'environnement ; design produit ; design textile, etc. 
ou au style (automobiles...). Or, chaque design concerne à la fois des desseins (conceptualisation, projet, stratégie) et leur mise en forme dans des dessins appropriés ; car les designs allient conceptualisation et intuition, sensible et intelligible, à l'opposé des classiques dichotomies philosophiques. Mais en tant que ressources stratégiques et créatives, ils procèdent bien du bricolage dont parlait Jean-Marie Floch pour les identités visuelles, la production de signifiant fonctionnant plus souvent selon l'intuition et l'expérimentation du travail d'atelier qu'avec le calcul ou la recherche de laboratoire'.

Les auteurs qui plaident pour un management du design ${ }^{2}$ l'ont montré aussi, ce n'est pas qu'un effet de style, une question de traitement décoratif de la surface ou une mise au goût du jour des objets. Si le design est une mise en forme, c'est dans tous les sens du terme forme, non-limitée au registre visuel. L'expressivité de cette forme ne saurait donc être pensée en dehors des concepts et des stratégies qui la motivent, ce qui nous éloigne alors de la conception de Kant.

Aujourd'hui, une organisation ne peut plus se contenter des apparences, du style, du look. La qualité est systémique, pas épidermique. L'esthétique -notamment des marques- se construit d'abord sur un territoire de valeurs, sur la pensée politique et stratégique d'une communauté sociale, sans négliger pour autant les modalités d'appropriation des objets sensibles. Un designer ne peut se limiter à la conception fonctionnelle d'un produit, à le rendre plaisant ou utile, ce qui est encore une conviction très partagée ${ }^{3}$.

Ainsi, le design est généralement compris comme une double activité de "résolution de problème" et de "création esthétique"4, ce qui laisse supposer la non-causalité réciproque entre ces deux derniers termes, comme pour esthétique et originalité. Le design n'est pas de l'art (il n'est pas détaché), ni de la science (il ne peut démontrer), même s'il se nourrit effectivement des démarches spécifiques à ces domaines. Tout ce qui relève du design n'est pas forcément esthétique, et ce qui est perçu comme esthétique n'est pas obligatoirement du design. Par exemple, la signalétique et ses pictogrammes procèdent d'une recherche de lisibilité et de visibilité qui ne vise pas en premier

1 J.-M. FLOCH, Identités visuelles, Paris, PUF, 1995.

$2 \mathrm{Br}$. Borza de Mozota, op. cit.; Ph. RAsQuinet et M. BRun, L'identité visuelle de l'entreprise, Paris, Éd. d'Organisation, 1996.

3 Chr. Chaptal de Chanteloup, Design : la stratégie du profit et du plaisir, Paris, Dunod, 1993.

4 Br. BORZA de MOZOTA, op. cit. 
lieu un effet esthétique, mais l'écriture spécifique qui en résulte, voir les jeux olympiques de Tokyo (1964) et de Munich (1972), fonde une esthétique du pictogramme désormais internationalement instituée.

L'esthétique des designs doit faire face à des oppositions artificielles entre réduction fonctionnelle et enrichissement symbolique. Pourtant, la forme d'un objet matériel propose plus qu'une fonction ; elle apporte à un utilisateur, exigeant en signification, des valeurs de reconnaissance et d'identification dépassant largement l'utilitaire. Les designs créent une synergie entre système et esthétique, ils font jouer ensemble les composantes imaginaires, structurelles, fonctionnelles et sensibles d'objets manufacturés en relation directe avec des usages. Les stratégies esthétiques relèvent ainsi de démarches de design global qui exigent une prise de conscience et une volonté spécifiques, car on ne peut faire du design sans le savoir. Or la question n'est pas forcément traitée de cette manière par une direction appropriée dans les organisations ; l'esthétique est encore souvent comprise comme une dépense et pas un investissement, sans doute en raison des connotations artistiques persistantes que le terme véhicule. Par exemple, une conception usuelle admet logiquement que la séduction, l'attractivité ou l'ergonomie des sites web (leurs interfaces) puissent être directement en rapport avec leur design, mais seulement compris comme traitement de surface, pas intervention sensible sur le système...

L'esthétique est aussi une compétition conceptuelle et créative pour ceux dont c'est le métier. Avant de faire commerce de leur savoir-faire esthétique, les designers doivent d'abord en passer par les rituels des appels d'offres ou des concours destinés à les sélectionner. Ils s'affrontent dans la férocité esthétique de ces joutes modernes à coup de concepts et de formes, de créativité et de consensus, afin de ravir les faveurs de la belle entreprise qui souhaite qu'on lui refasse une beauté. À cette occasion, les designers endossent volontiers les atours et les missions du chevalier blanc... Mais face aux rouages complexes et aux paramètres souvent obscurs de la décision, ils peuvent être tentés par les compromis trop consensuels, les renoncements et les complaisances éthiques qui favorisent les esthétiques banalisées des effets de mode.

Si les designs sont une pragmatique du changement dans les organisations, l'esthétique y contribue comme une pragmatique $d u$ sensible. Cette dimension est un espace tampon, un topos d'incertitude et d'humanité qui permet de contourner les rationalités dominantes, de sémiotiser autrement les relations entre sujets, objets et 
environnements, de donner des supports aux émotions, aux affects et au plaisir qui sont essentiels dans les constructions de relations. Dominique Blin a suggéré la capacité des emblèmes d'entreprise à "mancuvrer du plaisir" et pas simplement identifier". On sait que l'esthétique des logotypes est soigneusement entretenue pour plaire et être à la mode (on parle d'ailleurs de lifting... ). Le plaisir esthétique serait donc bien là, au cour de l'organisation mais il ne serait pas qu'un adjuvant stratégique ou un cosmétique de vente. Loin de toujours coïncider avec des valeurs déclarées, les systèmes graphiques, par le fait même qu'ils s'adressent aux facultés sensibles, offrent d'évidentes marges de manœuvre perceptives et créatives qui en font de précieux médiateurs du changement. Il est plus facile de montrer ce que l'on ne peut dire.

Le design est donc avant tout une démarche conceptuelle et créative qui affirme sa dimension systémique et globale à la fois dans le process et dans l'esthétique, il développe sa force stratégique dans le sensible comme dans l'intelligible. Reste à prendre en compte sa part technologique, essentielle aux esthétiques d'aujourd'hui.

\section{Tic et esthétique}

Les chercheurs s'intéressent beaucoup aux technologies dans les Sic, mais bien peu à leurs dimensions esthétiques au sens large. L'approche sensible se réduit un peu vite à des commentaires formels d'interfaces graphiques. Or, comment peut-on penser les usages sans leurs modalités sensibles, corporelles et sensorielles ? Ces relations sont souvent rabattues sur des critiques fonctionnelles de cohérence, de charte graphique, d'ergonomie, etc. ; mises en conformité avec les métaphores d'usage qui contaminent l'approche scientifique (très commodes mais peu discutées) ; soumises à des grilles de critères formels ; ou encore vues à travers des "codes esthétiques" sommaires qui limitent l'analyse à du commentaire de signifiant.

En production, les procédures et les langages numériques favorisent l'accessibilité à la création assistée et à ses esthétiques natives (induites par les logiciels) ; on parle d'animation Flash, de pages XML, d'effets Photoshop... La facilité apparente du numérique

1 D. BLIN, "Les emblèmes d'entreprises : la part du non verbal", in H. HotieR (dir.), Non verbal et organisation, Paris, GRECO-L'Harmattan, 2000. 
tend à superficialiser les interventions sensibles en les enfermant dans des effets qui ne peuvent que biaiser ou perturber les expressions comme les impressions sensibles et donc les analyses qui en sont faites. Goody ${ }^{1}$ ne démentirait sans doute pas cette modélisation de notre perception sensible et de nos écritures par le support... Toutefois, les modèles hérités de l'esthétique du livre et des traditions éditoriales papier ne sont pas pour autant évacués dans les processus de conception et de lecture. De plus, les "TIC" ne refondent pas forcément les critères du jugement et du goût qui restent ceux des acteurs de la commande, de la création et de la décision, situés dans leurs environnements et leurs histoires. Les économies du signe qui s'y déploient sont pourtant bien différentes ${ }^{2}$. Il n'est donc pas étonnant d'observer de très grands écarts entre les références esthétiques et les modèles convoquées en conception (les pratiques et cultures des créatifs) et celles sur lesquelles se fonde la décision (les cultures générales et habitus des managers), sans oublier ce que mobilisent les destinataires pour produire le sens.

\section{Les raisons du sensible}

De l'ordre de la séduction, la raison sensible est selon Caune ${ }^{3}$ une manière d'appréhender globalement les phénomènes, de participer à la communauté. Chaque organisation a de bonnes raisons d'utiliser les voies sensibles pour réaliser ses projets, on peut donc parler d'esthétiques instrumentalisées quand toute production sensible est astreinte à des objectifs et des logiques de pouvoir.

Le marketing rationalise l'esthétique avec ce qui marche, ou ce qui a de l'impact, le jugement étant centré sur les effets, l'accroche, en référence à des normes d'usage et des critères du beau issus d'un consensus forgé par l'habitude. Ce qui est beau est ce qui est conforme aux doctrines en vigueur dans les pratiques du métier, et donc supposé plaire (efficacement) à tous. Les certitudes sur les couleurs (le jaune est ceci, le vert est cela...) et les crispations sur les

1 J. GoOdy, La raison graphique, la domestication de la pensée sauvage, Paris, Éd. de Minuit, 1979.

2 E. Souchier et Y. Jeanneret, Pour une poétique de l'écrit d'écran, Xoana, JM Place, 1999.

3 J. CAUNE, Esthétique de la communication, op. cit. 
chartes graphiques ${ }^{1}$, vues comme de simples catalogues de signifiants ou d'effets décoratifs, laissent parfois penser que l'esthétique serait un système de recettes rationalisables. Il suffirait de mettre telle forme ou telle couleur pour obtenir tel effet, d'autant plus que les catégories socio-professionnelles tendent à se voir attribuer diverses esthétiques par l'enregistrement des usages, obéir à des codes et des univers graphiques spécifiques (et si rassurants...), que les consultants et parfois les chercheurs entérinent trop souvent sans recul (notamment dans les analyses de sites web). Les manières de montrer se codifient alors autant que les manières de dire, ce qui restreint la pertinence possible des expressions sensibles et biaise toute recherche en ce domaine.

À propos des usages contemporains, Parret parle des sous-catégories esthétiques répandues -moins nobles que le sublime, le beau ou le tragique-comme le joli, l'élégant ou le gracieux ${ }^{2}$. Ces considérations esthétiques courantes dans les organisations sont en effet peu exigeantes, plus faciles à mettre en œuvre, mais ce ne sont donc pas des esthétiques pleines. Dans ces problématiques du sens commun, du "bon goût", on retrouve le rapport tendu entre normes, contraintes et règles dont parle Parret pour les discours.

L'esthétique a donc ses raisons qui s'expriment en termes de valeur, mais elle est toujours soumise au jugement hâtif. Une stratégie de création cherche évidemment à produire des écarts sensibles et à maîtriser leur acceptabilité, mais en se nourrissant des contraintes et de ses environnements, elle propose aussi de nouvelles configurations qui peuvent à leur tour devenir des normes ou des styles dominants (voir les graphistes N. Brody ou D. Carson...).

1 La notion de charte graphique (ou livre de normes) est par définition normative, mais elle ne définit certainement pas l'esthétique de l'organisation, seulement quelques identifiants et leurs règles d'application sur tous les supports de communication. Le mot est sans doute dévoyé de son principe initial par les pratiques du web design car c'est un guide de conduite, pas un catalogue d'effets à coller partout, mais surtout, il faut savoir l'interpréter. Beaucoup de professionnels lui préfèrent le terme de charte créative qui, en ne définissant que des valeurs, qualifie une programmation visuelle très ouverte sur de multiples combinatoires et variantes graphiques qui enrichissent le système, une manière de dire et pas un raidissement sur des signifiants définitifs.

2 H. PARRET, op. cit. 


\section{Éthique et esthétique}

Kant définissait le beau comme référence pour la vie sociale, toute intervention sur le sensible aurait alors des dimensions morales. Mais si les rapports entre esthétique et éthique semblent étroits, on ne peut néanmoins les confondre. À travers des productions d'objets fonctionnels et signifiants, les designs tendent à transformer les contraintes, proposer une manière de voir les choses et stimuler une consommation différente. En s'intéressant aux relations entre des objets et des sujets dans leurs environnements cognitifs et sociaux, ils manifestent une conception environnementale, donnent un point de vue sur le devenir humain. Ainsi, ces démarches procèdent d'une attitude réformatrice, liée à une demande de changement ou de nouveauté, ce qui fonde leurs éthiques et idéologies, au risque de les amener parfois à s'enfermer dans les doctrines. Quoi qu'ils fassent, les designs articulent toujours des idéologies dans le sensible...

$\grave{A}$ travers leurs diverses orientations (consommation, communication, industrie, mobilier, réseaux, écologie, environnement, urgence...), les designs veulent intervenir sur le monde et manifester une responsabilité sociale. Avec plus ou moins de conviction marchande ou non-marchande, ils orientent le marché des signes vers la qualité, le respect, la communauté, l'émotion, la responsabilité, etc., valeurs qui nourrissent des esthétiques opérationnelles. Ils contribuent ainsi à l'identification $a ̀$ et $d e$ la société à travers ses objets, ce qui alimente une archéologie du futur faite de traces esthétiques et techniques. Ainsi, l'organisation doit concilier les valeurs socialement partagées qu'elle proclame esthétiquement, avec les argumentations exigées par le marché. Ces problématiques sont celles des médiations visuelles d'aujourd'hui dans un environnement économique qui ne peut plus ignorer les modalités de consommation esthétique-éthique voulues par les humains dans les sociétés modernes.

\section{Vers une pragmatique du sensible}

L'esthétique de l'organisation se crée et s'analyse, pour partie, à travers les designs qui lui donnent une forme visuelle. Cette mise en 
œuvre systémique et stratégique de l'esthétique est l'une des modalités sensibles de la médiation. L'expérience sensible devrait être un objet de recherche plus affirmé pour les Sic car elle concerne tout ce que font les acteurs de et dans la médiation, elle fait système dans les organisations. Cette pragmatique du sensible, qui opère dans des situations et des environnements de communication de tous ordres, concerne la création et les usages des signes, objets et discours -plus dans ce qu'ils montrent que dans ce qu'ils disent- mais s'intéresse surtout à la manière dont ils touchent ou émeuvent. La démarche gagne aussi à repérer les intentions des acteurs de la production, les stratégies institutionnelles, éditoriales et auctoriales qui pilotent les objets analysés ainsi que le suggère Baxandall ${ }^{1}$ afin de mieux comprendre les choix dont ils procèdent et les raisons de leurs mises en actes. Si pour Parret "l'objet pragmatique (...) est constitué comme un réseau de raisons", elles ne se livrent pas d'elles-mêmes². L'analyse de la dimension esthétique oblige à la sortir des conceptions classiques, des a priori et des idées reçues, à la protéger des jugements, afin de l'appréhender comme dispositif de médiation avec ses valeurs, modèles, processus, normes, répertoires, acteurs, etc., dans un cadre systémique qui reconnaîtrait une certaine logique à l'expression sensible. Malgré cela, une bonne partie des dimensions sensibles se dérobera toujours (et heureusement) au contrôle rationnel, ce qui laisse une part d'humain et une marge de manœuvre substantielle -en réception comme en production- dans des stratégies et des attentes parfois trop programmées et aseptisées.

1 M. BAXANDaLl, Les formes de l'intention, Nîmes, Chambon, 1985.

2 H. PARRET, op. cit. 